\title{
Search for isomeric transitions in fission products around mass number 150
}

\author{
Y. Kojima ${ }^{1, \text { a }}$, M. Shibata ${ }^{2}$, A. Taniguchi ${ }^{3}$, A. Murataka ${ }^{1}$, K. Ota ${ }^{1}$, and K. Shizuma ${ }^{1}$ \\ ${ }^{1}$ Graduate School of Engineering, Hiroshima University, Higashi-Hiroshima 739-8527, Japan \\ 2 Radioisotope Research Center, Nagoya University, Nagoya 464-8602, Japan \\ 3 Research Reactor Institute, Kyoto University, Kumatori 590-0494, Japan
}

\begin{abstract}
A $\beta$-gated $\gamma$-ray and internal conversion electron measurement system for identifying isomers has been installed to the isotope separator on-line at the Kyoto University Research Reactor. On-line measurements for mass number $A=93$ nuclides clearly showed that it had sufficient ability to distinguish isomeric transitions from those following $\beta$-decay. Using this equipment, an isomeric state in ${ }^{148} \mathrm{Pr}$ was successfully observed at $76.8 \mathrm{keV}$, and one in ${ }^{151} \mathrm{Pr}$ at $35.1 \mathrm{keV}$. We also found a candidate of an isomeric transition in an $A=150$ nuclide produced by the thermal neutron-induced fission of ${ }^{235} \mathrm{U}$.
\end{abstract}

\section{Introduction}

Experimental studies on isomers give useful information for nuclear science and technology. Existence of isomers directly relates to nuclear configurations. Thus, properties on single particle orbitals are obtained from such experimental data on isomers as spins, parities, level energies and transition probabilities. These data will help to understand nuclei in detail and to improve nuclear theories. In the viewpoint of applications, on the other hand, the data for each isomer are important to develop reliable nuclear databases. For example, decay heat libraries of fission products are one of the most important data in nuclear engineering, and they are evaluated through summation calculations. In this method, the total energy is calculated as the sum of energies released from individual $\beta$-decaying nuclides. Nuclear model calculations are also taken into account in the evaluations to compensate the lack of experimental data. The decay heat calculated by the summation method is fairy consistent with the integrated measurements for various fissionable nuclides [1,2]. However, there remains differences between the calculations and experiments in short cooling times of 1-2000 s after a burst fission. This small but systematic discrepancy would be partly due to a scarcity of experimental data on isomers [3].

In general, it is difficult to measure transitions from isomers (isomeric transitions) in fission products. This is because production yields of isomers are usually small and isomeric transitions are hidden in spectra measured for fission products. In these spectra, transitions following $\beta$-decay are strongly observed in a wide energy range. To overcome this difficulty, measurements of isomeric transitions were performed using $\beta$-gated $\gamma$-rays and internal conversion electrons (ICEs) detection system at an on-line isotope separator (ISOL). The ISOL leads nuclides having a mass number of interest to a low-background measuring port, and the $\beta$-coincident measurements unambiguously distinguish isomeric transitions from other transitions due to $\beta$-decay. In this contribution, we present the experimental setup together with its performance, and show results on measurements for ${ }^{148 \mathrm{~m}} \mathrm{Pr},{ }^{151 \mathrm{~m}} \mathrm{Pr}$ and a mass number $A=150$ nuclide. For ${ }^{148} \mathrm{Pr}$, the existence

\footnotetext{
${ }^{a}$ Presenting author, e-mail: ykojima@hiroshima-u.ac.jp
}

of isomers has been known from two different half-lives: $2.29 \mathrm{~min}$ for the $I^{\pi}=1^{-}$ground state, and $2.01 \mathrm{~min}$ for the $I=$ (4) level [4]. However, the energy difference between the two states is unknown because the isomeric transition has not been observed. For neutron-rich $A=150$ and 151 isotopes, no isomers has been reported yet $[5,6]$.

\section{Experiment}

\subsection{Source preparation}

Radioactive nuclei were produced by the thermal neutroninduced fission of ${ }^{235} \mathrm{U}$, followed by the on-line isotope separator at the Kyoto University Research Reactor (KURISOL) [7]. A 93\%-enriched $50 \mathrm{mg}{ }^{235} \mathrm{UF}_{4}$ target was irradiated in a through-hole of the 5-MW reactor. The thermal neutron flux was $3 \times 10^{12} \mathrm{n} / \mathrm{cm}^{2} \mathrm{~s}$ at the source position. The fission products were thermalized in the target chamber, and transported to a surface-ionization-type ion source by a $\mathrm{He}-\mathrm{N}_{2}$ mixed gas-jet stream. After ionization, the radioactive ions were extracted, accelerated to $30 \mathrm{keV}$, and mass-separated with a resolution $M / \Delta M$ of approximately 600 . The massseparated nuclides were implanted into an aluminized Mylar tape in a computer-controlled moving tape system. The tape periodically transported the radioactive sources to a lead-shielded detector station.

\subsection{Detectors}

To identify isomeric transitions among intense radiations originating from $\beta$-decay, $\beta$ - $\gamma$ and $\beta$-ICE coincidence measurements were performed. Most of $\gamma$-rays and conversion electrons depopulate short-lived excited states $\left(T_{1 / 2} \ll 1 \mu \mathrm{s}\right)$ following $\beta$ decays, so that they will be coincident with $\beta$ rays. On the contrary, isomeric transitions will not be observed in the $\beta$-gated spectra because they are populated from long-lived levels. Detectors used for the experiments were as follows: a $30 \%$-type HPGe detector (ORTEC GMX) or a short coaxial 
Ge detector (ORTEC LO-AX, $52 \mathrm{~mm}^{\phi} \times 20 \mathrm{~mm}^{t}$ ) for $\gamma$-rays, a cooled $\mathrm{Si}(\mathrm{Li})$ detector $\left(25 \mathrm{~mm}^{\phi} \times 6 \mathrm{~mm}^{t}\right)$ for ICEs, and a $1 \mathrm{~mm}$ thick plastic scintillator $(80 \mathrm{~mm} \times 90 \mathrm{~mm})$ for $\beta$ rays. The plastic scintillator was installed in the ISOL vacuum chamber and positioned at $1 \mathrm{~mm}$ from the source. The solid angle subtended by the scintillator was approximately $45 \%$. To absorb conversion electrons, the plastic scintillator was covered by a $0.2-\mathrm{mm}$-thick aluminum foil, which corresponds to a range for $0.2-\mathrm{MeV}$ electrons. The $\mathrm{Si}(\mathrm{Li})$ detector was fixed in a small vacuum chamber, which was separated from the ISOL chamber by a $0.5 \mu$ m thick polyester film to prevent residual vapors from being trapped on the cooled surface of the detector. A source-to-detector distance was $13 \mathrm{~mm}$ for the $\mathrm{Si}(\mathrm{Li})$, and $9 \mathrm{~mm}$ for the HPGe detector. Conventional NIM modules were used for the coincidence experiments. A time range of the time-to-amplitude converters (TAC) was set to $2 \mu \mathrm{s}$, and a coincidence time of the data acquisition system was $5 \mu \mathrm{s}$. Therefore, transitions from excited levels having a half-life $T_{1 / 2} \lesssim 10 \mu$ s were observed in this $\beta$-gated coincidence system; For radiations depopulating levels with $T_{1 / 2}>$ $10 \mu \mathrm{s}$, only an extremely small part $(<10$ were fired by the TAC gate.

A singles and $\beta$-gated spectrum for each detector were taken in a spectrum-multi-scaling (SMS) mode to deduce halflives. In the SMS, the counting period in each tape cycle was divided into 16 time bins.

The energy and efficiency calibration were performed using standard $\gamma$-ray sources of ${ }^{133} \mathrm{Ba},{ }^{137} \mathrm{Cs},{ }^{60} \mathrm{Co}$ and ${ }^{152} \mathrm{Eu}$. Coincidence summing effects were taken into account in deducing peak counts. Calibration for the $\mathrm{Si}(\mathrm{Li})$ detector was made on-line using electrons from mass-separated ${ }^{93 \mathrm{~m}} \mathrm{Y}$ and ${ }^{146} \mathrm{La}$. Monte Carlo calculations by the EGS4 code [8] were also used to inter- and extrapolate an energy dependence of the detection efficiencies. More detailed description on the detection system is given in refs. $[9,10]$.

\subsection{Measurements}

Performance of the $\beta$-coincident system, that is, a capability to distinguish isomeric transitions from the other transitions following $\beta$ decays was studied through an on-line measurements for mass-separated $A=93$ nuclides. This mass fraction contained $\beta$-decaying isotopes ${ }^{93} \mathrm{Rb}\left(T_{1 / 2}=5.8 \mathrm{~s}\right)$ and ${ }^{93} \mathrm{Sr}$ $\left(T_{1 / 2}=7.4 \mathrm{~min}\right)$, and the known isomer ${ }^{93 \mathrm{~m}} Y\left(T_{1 / 2}=0.82 \mathrm{~s}\right)$. Thus, $A=93$ nuclides were suitable to check and demonstrate the feasibility of the detection system. A tape cycle for this calibration experiment was $20 \mathrm{~s}$, and a measurement period was about $3 \mathrm{~h}$.

Next, search for isomeric transitions was performed for $A=148,150$ and 151 nuclides. In these experiments, the radioactive isotopes were mass-separated as a chemical form of a monoxide ion by injecting a small amount of $\mathrm{O}_{2}$ to the He- $\mathrm{N}_{2}$ gas stream. This is because the oxidation technique improves ionization efficiencies for lanthanides [11]. A tape cycle and a measurement period were as follows: $113 \mathrm{~s}$ and $18 \mathrm{~h}$ for $A=148,160 \mathrm{~s}$ and $21 \mathrm{~h}$ for $A=150,3.5 \mathrm{~s}$ and $182 \mathrm{~h}$ for $A=151$, respectively. Background spectra were also measured for $4 \mathrm{~h}$ when in operation of the reactor.
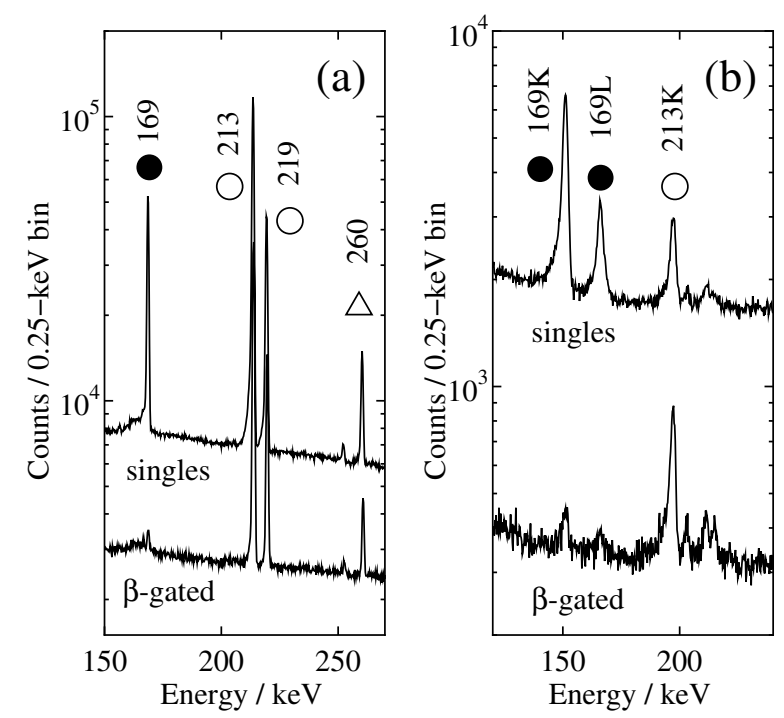

Fig. 1. (a) A singles and $\beta$-gated $\gamma$-ray, and (b) internal conversion electron spectrum for a mass fraction of $A=93$. Close circles represent ${ }^{93 \mathrm{~m}} \mathrm{Y}$, open circles ${ }^{93} \mathrm{Rb}$ and triangle ${ }^{93} \mathrm{Sr}$.

\section{Results and discussion}

\section{1 $A=93$, a calibration measurement}

A singles and $\beta$-gated spectrum for $\gamma$-rays and conversion electrons were presented in figure 1 . This clearly shows that the $169-\mathrm{keV}$ isomeric transition from ${ }^{93 \mathrm{~m}} \mathrm{Y}$ is greatly reduced in the $\beta$-gated spectrum while the other peaks following $\beta$ decay are strongly observed in both the singles and $\beta$-gated spectra. To evaluate the performance, we introduced a $\beta$ gate efficiency which was defined as a ratio between the peak count observed in the $\beta$-gated spectrum to that in the singles. As described in detail in our previous paper [10], the deduced efficiency was 0.016 (2) for the isomeric transition and $0.278(4)$ for those originating from $\beta$-decay. Thus, we conclude this detection system has an adequate ability to identify isomeric transitions in spectra measured for fission products.

\section{$3.2^{148 m} \mathrm{Pr}$}

Figure 2 shows an electron singles and a $\beta$-gated electron spectrum for a mass fraction of $A=148+16$. The 34.6and $70.8-\mathrm{keV}$ electron peaks were clearly observed in the singles, but highly reduced in the $\beta$-gated spectrum. Because the gate efficiency observed for the $35-\mathrm{keV}$ peak was $0.137(5)$ and approximately 0 for the $71-\mathrm{keV}$ electron, they were isomeric transitions. The energy difference of the two peaks was consistent with that of the electron binding energy of Pr: $B_{K}=42.0 \mathrm{keV}$ and $B_{L}=6.4 \mathrm{keV}$. In addition, the halflife observed for the $71-\mathrm{keV}$ peak agreed with the value for the high-spin isomer of ${ }^{148} \mathrm{Pr}$. Therefore, these peaks originate from the isomeric decay of ${ }^{148 \mathrm{~m}} \mathrm{Pr}$, namely, the $76.8-\mathrm{keV} K$ and $L$ conversion electrons. Here, we note that the $35-\mathrm{keV}$ peak was a multiplet of electrons from the isomeric decay of ${ }^{148 \mathrm{~m}} \mathrm{Pr}$ 


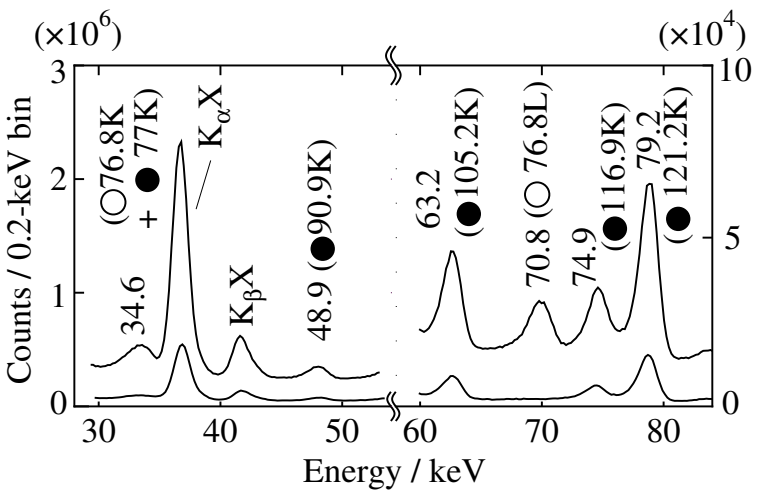

Fig. 2. A singles and $\beta$-gated conversion electron spectrum for a mass fraction of $A=148+16$. Open circles represent ${ }^{148 \mathrm{~m}} \mathrm{Pr}$ and closed circles ${ }^{148} \mathrm{Ce}$.

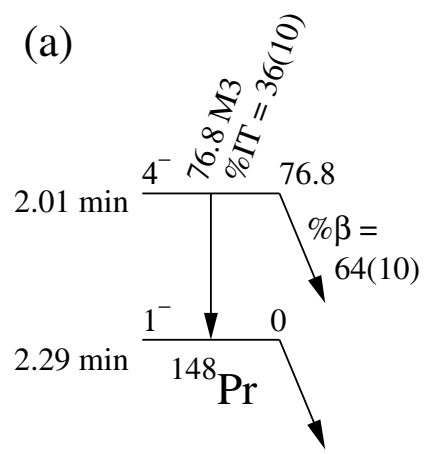

Fig. 3. (a) The decay scheme of ${ }^{148 \mathrm{~m}} \operatorname{Pr}$ and (b) ${ }^{151 \mathrm{~m}} \mathrm{Pr}$.

and those from the $\beta$-decay of ${ }^{148} \mathrm{Ce}$. It is a reason why the $\beta$-gate efficiency for the $35-\mathrm{keV}$ electron shows an inbetween value of 0.01 and 0.3 .

From an intensity ratio of the electron to the $\gamma$-ray peak counts and their detection efficiencies, $K$-conversion coefficient of 151(39) was deduced for the 76.8-keV $\gamma$ transition. This value well agrees with the theoretical value of 170 for $M 3$ [12]. Because the ground state of ${ }^{148} \mathrm{Pr}$ is a $1^{-}$state [4], the spin-and-parity of $4^{-}$was unambiguously assigned to the 76.8-keV isomeric level (fig. 3(a)).

From the systematics of low-lying levels in neighboring $N=89$ isotones and $Z=59$ isotopes, and the GallagherMoszkowski rules, the configurations for the two states were proposed as follows: $\pi 3 / 2^{+}$[411] $v 5 / 2^{-}$[523] for the $1^{-}$ground state, and $\pi 5 / 2^{+}[413] v 3 / 2^{-}[532]$ for the $4^{-}$isomer. More detailed description is given in ref. [9].

\section{3 $A=150$ nuclide}

Figure 4 shows a low-energy part of $\gamma$-ray spectra measured for $A=150+16$. While most of the peaks were seen in both the single and the $\beta$-gated spectra, a $65.3-\mathrm{keV}$ peak was observed only in the singles. This implies that the $65-\mathrm{keV}$ $\gamma$-ray is a candidate of an isomeric transition from an unknown isomer having a mass number of 150 . Unfortunately, we could not deduce the origin, that is, the atomic number of the isomer. Experimental results that we obtained until now are

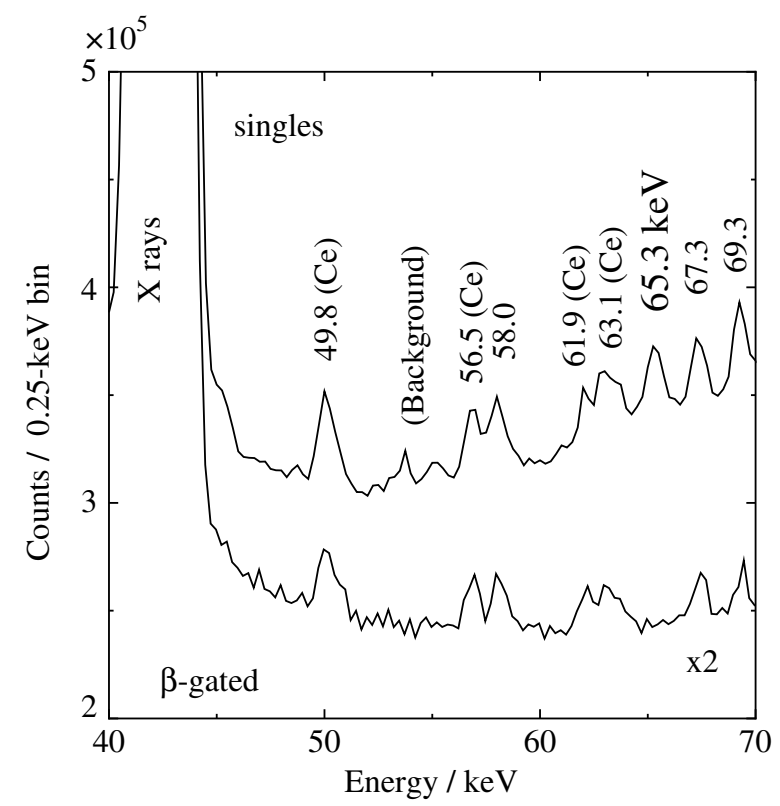

Fig. 4. A singles and $\beta$-gated $\gamma$-ray spectrum observed for a mass fraction of $A=150+16$.

as follows. First, the $65-\mathrm{keV} \gamma$ ray is not coincident with any $\beta$ rays and conversion electrons although statistics may not be sufficient. Second, the $65-\mathrm{keV}$ peak is observed only in the first 10 -s interval of the time-dependent $\gamma$-ray singles, so that the half-life is much shorter than $10 \mathrm{~s}$. Further experiments are planned in near future.

\section{$3.4^{151 \mathrm{~m}} \mathrm{Pr}$}

We have reported a partial decay scheme of ${ }^{151} \mathrm{Ce}$ at the ND2001 conference [13]. In that study, the 35.1-keV $\gamma$ ray, which was most intensely observed, was not included in the decay scheme because no $\gamma-\gamma$ coincidence relations were observed for it. After that, the $\beta$-gate efficiency for the $35-\mathrm{keV} \gamma$-ray was found to be 0.021 (3) from additional $\beta-\gamma$ coincidence measurements. Thus, the $35-\mathrm{keV} \gamma$-ray was assigned as the transition from the $35.1-\mathrm{keV}$ isomeric level in ${ }^{151} \mathrm{Pr}$ to the ground state. As shown in figure $3(\mathrm{~b})$, the spin-parity of $7 / 2^{+}$was tentatively proposed for ${ }^{151 \mathrm{~m}} \mathrm{Pr}$ from a small $\log f t$ value to this level [10]. Note, this evaluation was based on the spin-parity assignment of $\left(5 / 2^{+}\right)$for the ground state of ${ }^{151} \mathrm{Ce}$ [14], that of $(3 / 2)^{-}$for ${ }^{151} \operatorname{Pr}[15]$, and $\beta$-feeding intensities to excited levels in ${ }^{151} \mathrm{Pr}$ observed in our work.

It is apparent that the half-life of this isomeric state is longer than $\sim 10 \mu$ s because the $35-\mathrm{keV} \gamma$-ray is not coincident with $\beta$ rays. However, The definite value could not be obtained owing to the following reason. The decay curve of the $35-\mathrm{keV}$ $\gamma$-ray showed a straight line in a semi-log plot. In other words, the decay curve did not indicate a growth-and-decay property. The "half-life" deduced from a single-component exponential curve was $1.79(25) \mathrm{s}$, and it well agreed with the $\beta$-decay half-life of ${ }^{151} \mathrm{Ce}(1.76(6) \mathrm{s})$. This means that ${ }^{151} \mathrm{Ce}$ and ${ }^{151 \mathrm{~m}} \mathrm{Pr}$ are in radioactive equilibrium. Consequently, the half-life of ${ }^{151 \mathrm{~m}} \mathrm{Pr}$ is greatly shorter than $1.76 \mathrm{~s}$. 


\section{Conclusions}

A detection system to search new isomers in fission products of ${ }^{235} \mathrm{U}$ has been installed to the on-line isotope separator at Kyoto University Research Reactor. It is based on the $\beta-\gamma$ and $\beta$-electron coincidence measurements, and has a sufficient ability to identify isomeric transitions. Using this apparatus, we successfully observed isomers in ${ }^{148} \mathrm{Pr}$ and ${ }^{151} \mathrm{Pr}$, and newly found a candidate of an isomeric transition from a nuclide having a mass number of 150 .

This work was performed under the Research Collaboration Program of Research Reactor Institute, Kyoto University. One of the authors (YK) acknowledges Electric Technology Research Foundation of Chugoku for its financial support.

\section{References}

1. J.K. Dickens, T.A. Love, J.W. McConnell, R.W. Pelle, Nucl. Sci. Eng. 74, 106 (1980).

2. N. Hagura, T. Yoshida, T. Tachibana, J. Nucl. Sci. Technol. 43, 497 (2006).

3. T. Yoshida, T. Tachibana, F. Storrer, K. Oyamatsu, J. Katakura, J. Nucl. Sci. Technol. 36, 135 (1999).
4. M.R. Bhat, Nucl. Data Sheets 89, 797 (2000).

5. E. derMateosian, J.K. Tuli, Nucl. Data Sheets 75, 827 (1995).

6. B. Singh, Nucl. Data Sheets 80, 263 (1997).

7. A. Taniguchi, K. Okano, T. Sharshar, Y. Kawase, Nucl. Instrum. Meth. A 351, 378 (1994).

8. W.R. Nelson, H. Hirayama, D.W.O. Rogers, The EGS4 code system, SLAC-R-265 (1985).

9. Y. Kojima, A. Taniguchi, M. Shibata, E. Oyama, T. Nishimura, K. Shizuma, Y. Kawase, Eur. Phys. J. A 19, 77 (2004).

10. Y. Kojima, M. Shibata, A. Taniguchi, Y. Kawase, R. Doi, A. Nagao, K. Shizuma, Nucl. Instrum. Meth. A 564, 275 (2006).

11. Y. Kawase, K. Okano, Nucl. Instrum. Meth. B 37-38, 116 (1989).

12. F. Rösel, H.M. Fries, K. Alder, HC. Pauli, At. Data Nucl. Data Tables 21, 91 (1978).

13. Y. Kojima, M. Shibata, A. Taniguchi, Y. Kawase, T. Hanafusa, E. Yoshida, K. Shizuma, in Proceedings of the International Conference on Nuclear Data for Science and Technology, Tsukuba, 2001, edited by K. Shibata (Atomic Energy Society of Japan, 2002), p. 489.

14. F. Hoellinger, N. Schulz, J.L. Durell, I. Ahmad, M. Bentaleb, M.A. Jones, M. Leddy, E. Lubkiewicz, L.R. Morss, W.R. Phillips, A.G. Smith, W. Urban, B.J. Varley, Phys. Rev. C 56, 1296 (1997).

15. M. Shibata, T. Ikuta, A. Taniguchi, A. Osa, A. Tanaka, H. Yamamoto, K. Kawade, J.-Z. Ruan, Y. Kawase, K. Okano, J. Phys. Soc. Jpn. 63, 3263 (1994). 